\title{
El Debate Parlamentario sobre Inmigración y Salud en España
}

\section{The Parliamentary debate on immigration and health in Spain}

\author{
Andrés A. Agudelo-Suárez ${ }^{1,2}$, Diana Gil-González ${ }^{2}$, Carmen Vives-Cases² \\ y Elena Ronda-Pérez ${ }^{2}$
}

1Facultad de Odontología. Universidad deAntioquia.Medellín-Colombia.

agudeloandres@odontologia.udea.edu.co

2 Área de Medicina Preventiva y Salud Pública. Universidad de Alicante. Alicante. España. diana.gil@ua.es, carmen.vives@ua.es, elena.ronda@ua.es

Recibido 12 Diciembre 2008/Enviado para Modificación 5 Julio 2009/Aceptado 10 Julio 2009

\section{RESUMEN}

Objetivo Describir y analizar el debate parlamentario español sobre inmigración y salud. Métodos Búsqueda sistemática de las iniciativas parlamentarias (IP) sobre inmigración e inmigración y salud desde el inicio del periodo democrático español en 1979 hasta 2007. Se aplicó un protocolo de recogida de información para identificar las características del debate parlamentario y los principales temas en las IP relacionadas con inmigración y salud. Se calcularon la proporción de IP de inmigración sobre el total de iniciativas y la proporción de población inmigrante sobre la población española por cada año de estudio.

Resultados Se identificaron 4022 IP sobre inmigración en el periodo estudiado. Se destaca la generación de datos estadísticos sobre aspectos que afectan a la población inmigrante (57,2\%). En 116 IP sobre inmigración y salud, destaca la discusión sobre estrategias de atención sanitaria $(25 \%)$ y el acceso a servicios de salud $(24,1 \%)$. El 94 $\%$ de estas IP fueron preguntas correspondientes a la función de control del gobierno. En 113 IP (97,4 \%) sobre inmigración y salud no se tomaron decisiones. Junto a la presencia cada vez mayor de población inmigrante por año, no se observan cambios notorios en las IP sobre inmigración hasta el año 1996 y en inmigración y salud hasta 1999.

Conclusión El debate sobre inmigración y salud es heterogéneo. Seria conveniente fomentar la discusión sobre estrategias para el fomento de la salud basadas en las necesidades de la población inmigrante.

Palabras Clave: Migración internacional, formulación de políticas, políticas, planificación y administración en salud, poder legislativo, España (fuente: DeCS, BIREME).

\section{ABSTRACT}

Objective Describing and analysing the Spanish Parliamentary debate on immigration and health.

Methods A systematic search regarding parliamentary initiatives (PI) on immigration and immigration and health was conducted from the beginning of the Spanish democratic period (1979 to 2007). A protocol for collecting information was used to identify the 
parliamentary debate's main characteristics and the main topics related to PI concerning immigration and health. The PI immigration rate was calculated regarding the total rate of $\mathrm{PI}$ about the immigrant population based on the Spanish population per year.

Results 4,022 PI concerning "immigration" were identified. The main content concerned statistical information about the immigrant population (57.2\%). $116 \mathrm{PI}$ about "immigration and health" were analysed. The most frequently recurring topics were health-care strategies (25\%), health-service access (24.1\%) and epidemiological information (19.8 $\%)$. Most PI concerned questions related to the function of government control (94\%). No decisions were taken in $113 \mathrm{PI}(97.4 \%)$. Immigrant population rates increased per year; however, notable changes in PI regarding immigration were not observed until 1996 and PI about immigration and health until 1999.

Conclusion The immigration and health debate on the parliamentary agenda is heterogeneous. It would seem necessary to increase parliamentary debate about strategies and action for promoting immigrant-based needs in health.

Key Words: Emigration and immigration, policy-making, health policy, planning and management, legislative power, Spain (source: MeSH, NLM).

a sociedad Española está experimentando un proceso de cambio como consecuencia del incremento de la población procedente de países en desarrollo. Según datos del Instituto Nacional de Estadística -2008-, la proporción de inmigrantes de países europeos no comunitarios, de Asia y Centro y Sudamérica con relación a la población total española, ha aumentado entre 1990 y 2007 de $0,9 \%$ al 7,7 \% (1). La gran mayoría de la población inmigrante tiene como objetivo la integración en el mercado de trabajo para mejorar sus condiciones de vida (2). Una gran proporción de esta población son Latinoamericanos. De acuerdo con el Observatorio permanente para la inmigración -2007-, la población con tarjeta de residencia en vigor proveniente de Latinoamérica es 1215351 (53,6\% mujeres), constituyendo el 30,5\% de todos los extranjeros residentes en España, y con una media de edad de 32 años (3).

Desde 1990 se ha prestado especial atención en el parlamento a la puesta en marcha de un plan para la integración social de la población inmigrante; también existen medidas legislativas llevadas a cabo por el gobierno español como la ley 8/2000 sobre derechos y libertades de los extranjeros en España (4). Los aspectos de mayor interés se han centrado en la necesidad de legislar y planificar el fenómeno migratorio (5), la regularización de inmigrantes para su inserción en el mercado laboral (6) y algunos debates puntuales sobre el acceso y la asistencia sanitaria (7). 
Una revisión de estudios científicos y de documentos institucionales en España muestra el enfoque que se ha desarrollado en torno a la inmigración y la salud. Por ejemplo, ha habido un interés por analizar las denominadas "enfermedades importadas" (8) de la población inmigrante, además de las consecuencias en la salud de su adaptación al país de destino (9). Otro ámbito de estudio ha sido la atención sanitaria (10), los factores que afectan el acceso a los servicios de salud (11), problemas específicos en niños (12) y en mujeres inmigrantes (13); y el análisis de condiciones laborales y de salud en población trabajadora (14).

Sin embargo, son escasos los estudios que abordan las decisiones políticas en el contexto parlamentario sobre inmigración y salud. Aunque en ciencias políticas, se han elaborado propuestas de análisis del discurso en debates e intervenciones parlamentarias sobre la inmigración en España (15). El parlamento por lo tanto, constituye un referente de análisis, teniendo como fuente de información el Diario de Sesiones del Congreso de los Diputados (16). Esta metodología ha sido utilizada para el análisis de otros temas en salud pública (17-19).

El objetivo del presente estudio es describir y analizar el debate parlamentario español sobre inmigración y salud (1979-2007).

\section{MÉTODOS}

Búsqueda sistemática y análisis de contenido cuantitativo (20) de las iniciativas parlamentarias (IP) en el periodo democrático español (1979-2007). La información se obtuvo de la página Web del Congreso de los Diputados (21).

Para la búsqueda de las IP sobre "inmigración" se utilizaron las siguientes palabras clave: inmigración, migración, inmigrantes. La información extraída fue clasificada en 9 bloques temáticos: 1) Acuerdos internacionales, 2) Medios de comunicación e información: campañas informativas y de opinión, 3) Educación: estrategias para garantizar el acceso a la educación formal y no formal, 4) Información estadística, 5) Estrategias de lucha contra la inmigración ilegal: campañas con el fin de evitar el flujo de personas sin permiso de visado y estrategias para prevenir población ilegal 6) Políticas y estrategias dirigidas a la población inmigrante: políticas de integración, y estrategias en el ámbito social y económico 7) Políticas específicas dirigidas a las mujeres inmigrantes: integración social, la violencia de género, entre otros, 8) Aspectos relacionados con la situación laboral de la población inmigrante: contratación de personal, y procesos de regularización de los permisos de residencia y trabajo, y 9) Salud: Condiciones y determinantes de la salud en 
hombres y mujeres inmigrantes. Se estableció un capítulo de otros temas que agrupó un considerable número de IP ampliamente heterogéneas relativas a sucesos concretos protagonizados por población inmigrante.

Posteriormente las IP incluidas en el apartado de salud fueron reclasificadas y se realizó una nueva búsqueda para IP sobre "inmigración y salud". Para ello se utilizaron las siguientes combinaciones: inmigración y salud; migración y salud; inmigrantes y salud. Esto permitió obtener 116 IP.

Se realizó un estudio descriptivo de las variables del protocolo utilizado para el análisis de contenido según el reglamento del Congreso Español $(17,21)$. Tipo de funciones que corresponden las iniciativas parlamentarias: Legislativa, control parlamentario del gobierno, orientación política, otras; Tipo de Iniciativa Parlamentaria: Preguntas al Gobierno, con respuesta escrita, orales o en pleno, Proposiciones de ley, no de ley, comparecencias, Iniciativas Populares, Interpelaciones urgentes, entre otras; Órgano donde se discute la IP: Pleno (del Congreso o del Senado), Comisión (del Congreso o del Senado); Comisión donde se discute la IP; Sexo del Interlocutor que promueve la IP; Grupo Parlamentario que promueve la IP: Socialista, Popular, Mixto, Izquierda Unida, entre otros; Toma de decisiones con impacto en las políticas: (aprobado con o sin modificaciones o no aprobado).

Las IP sobre "inmigración y salud" se agruparon en 9 temas: 1) Recursos disponibles para brindar atención sanitaria; 2) Requerimientos de datos de información epidemiológica; 3) Acceso a servicios de salud; 4) Atención en salud específica para mujeres embarazadas; 5) Salud laboral e inmigración- Prevención en riesgos laborales y su relación con la salud; 6) Estrategias para dar atención en salud; 7) Requerimientos de pruebas diagnósticas y medidas de protección contra enfermedades infecciosas provenientes de otros países; 8) Convenios interinstitucionales en salud; y 9) Requerimientos de formación de profesionales sanitarios en inmigración.

Por último, se calcularon proporciones entre el número de inmigrantes que proceden de países Europeos no comunitarios, Asia y Latinoamérica y la población total española por cada año del estudio. Las fuentes de información para este cálculo fueron los anuarios estadísticos y el padrón municipal (1). Se calcularon proporciones de IP sobre inmigración y el número total de IP producidas en el Congreso por cada año de estudio. En las IP sobre inmigración y salud se utilizaron números absolutos. 
Se utilizaron los programas MS- Excel y SPSS versión 14.0 para el análisis de los datos.

\section{RESULTADOS}

Se identificaron 4022 IP sobre inmigración en el periodo estudiado. Los temas más frecuentes fueron los requerimientos de estadísticas sobre población inmigrante en España y sus comunidades autónomas (57,2 \%), aspectos relacionados con la situación laboral de hombres y mujeres inmigrantes (7,5\%), políticas y estrategias generales dirigidas a esta población $(4,6 \%)$ y estrategias para contrarrestar la inmigración ilegal $(4,3 \%)$. La salud constituye un 2,9\% de todas las IP. Un 19,5\% del total se clasificaron como otros (Tabla 1).

Tabla 1. Bloques temáticos de las Iniciativas Parlamentarias en España sobre temas relacionados con inmigración (1979-2006)

\begin{tabular}{lcc}
\hline \multicolumn{1}{c}{ Bloques temáticos (Inmigración) } & \multicolumn{2}{c}{$\begin{array}{c}\text { Iniciativas Parlamentarias } \\
(1979-2006)\end{array}$} \\
& $N$ & $\%$ \\
\hline Información estadística & 2300 & 57,2 \\
Aspectos relacionados con la situación laboral de hombres y & & \\
mujeres inmigrantes & 300 & 7,5 \\
Políticas y estrategias dirigidas a la población inmigrante & 185 & 4,6 \\
Lucha contra la inmigración ilegal & 173 & 4,3 \\
Salud & 116 & 2,9 \\
Educación & 86 & 2,1 \\
Acuerdos internacionales & 43 & 1,1 \\
Medios de comunicación e información & 18 & 0,4 \\
Políticas específicas dirigidas a las mujeres inmigrantes & 15 & 0,4 \\
Otros temas (heterogeneidad temática. Sucesos concretos & & \\
relacionados a población inmigrante no incluidos en los temas & & 19,5 \\
anteriores) & 786 & 100 \\
\hline \multicolumn{1}{c}{ TOTAL } & 4022 & \\
\hline
\end{tabular}

Se identificaron 116 IP sobre inmigración y salud. La mayoría fueron debatidas en las dos últimas legislaturas $(87,1 \%)$. El $94 \%$ de las IP obedecen a la función de control parlamentario. El 86,2 \% de las IP se discutieron en pleno, y el 56,3\% de las IP debatidas en comisión tuvieron lugar en la Comisión de Sanidad y Consumo. El 88,8 \% de las iniciativas fueron preguntas con respuesta escrita, orales, en pleno o comisión. En el 97,4 \% de las IP no se tomaron decisiones políticas. El $44 \%$ de las IP fueron lideradas por hombres y el 74,2 \% por los grupos parlamentarios popular y socialista (Tabla 2).

En relación al contenido de las IP (Tabla 2), el 25 \% trataron sobre políticas y estrategias para asegurar la atención sanitaria a la población inmigrante por parte de las instituciones competentes. El $24,1 \%$ se debatieron en torno al tema del 
acceso a los servicios de salud por parte de la población inmigrante. El 19,8\% versaron sobre la necesidad de información estadística y epidemiológica. Un cuarto bloque temático destacable fue el correspondiente a la atención de las mujeres inmigrantes embarazadas $(12,1 \%)$.

La Figura 1 muestra la evolución de las IP por año y de la población inmigrante. Mientras se observó una presencia cada vez mayor de inmigrantes con relación a la población española, en las IP que abordan el tema de inmigración se presentaron cambios notorios después del año 1996, con las mayores frecuencias en los años 2003 y 2006. En las iniciativas que abordan el tema de inmigración y salud, se reportaron cambios notorios después del año 1999 y las mayores frecuencias en los años, 2002 y 2006.

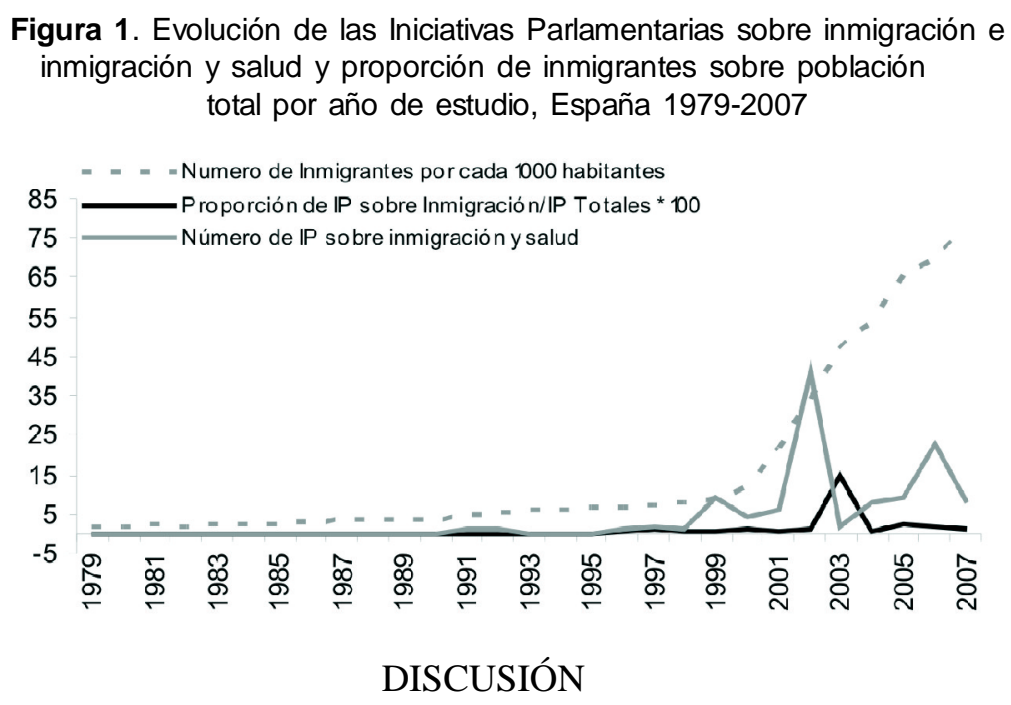

El debate político parlamentario español acerca de la inmigración se caracteriza fundamentalmente por la demanda y generación de información estadística. Por otra parte, el debate relativo a la inmigración y salud tiene como principal preocupación el acceso a los servicios de salud, las políticas y estrategias dirigidas a la atención sanitaria, y la generación de información epidemiológica. El interés político sobre el tema ha sido desigual en comparación con el incremento de los flujos migratorios. El debate se distingue por la realización de preguntas sin consolidar decisiones políticas. Aunque se reconoce la realización de diferentes proyectos de ley en el tema, la inmigración se encuentra en un momento de construcción política en el contexto parlamentario. 
Tabla 2. Características generales de las Iniciativas Parlamentarias en España sobre inmigración y salud, 1979-2007 ( $n=116)$

\begin{tabular}{|c|c|c|}
\hline Característica & $\mathrm{N}$ & $\%$ \\
\hline \multicolumn{3}{|l|}{ Legislatura } \\
\hline IV (1989-1993) & 2 & 1,7 \\
\hline VI (1996-2000) & 13 & 11,2 \\
\hline VII (2000-2004) & 53 & 45,7 \\
\hline VIII (2004-2007) & 48 & 41,4 \\
\hline \multicolumn{3}{|l|}{ Tipo de Función } \\
\hline Control & 109 & 94,0 \\
\hline Orientación Política & 5 & 4,3 \\
\hline Otras & 2 & 1,7 \\
\hline \multicolumn{3}{|l|}{ Tipo de Iniciativa } \\
\hline Pregunta al Gobierno con respuesta escrita & 95 & 81,9 \\
\hline Pregunta oral al Gobierno en Comisión o en pleno & 8 & 6,9 \\
\hline Proposición no de Ley en Comisión & 5 & 4,3 \\
\hline Comparecencia del Gobierno en Comisión & 4 & 3,4 \\
\hline Otras & 4 & 3,4 \\
\hline \multicolumn{3}{|l|}{ Órgano donde se discute la iniciativa } \\
\hline Pleno & 100 & 86,2 \\
\hline Comisión & 16 & 13,8 \\
\hline \multicolumn{3}{|l|}{ Comisión donde se discute la iniciativa $(n=16)$} \\
\hline Comisión de Sanidad y Consumo & 9 & 56,3 \\
\hline Comisión de Justicia e Interior & 3 & 18,7 \\
\hline Comisión de Trabajo y Asuntos Sociales & 2 & 12,5 \\
\hline Otras & 2 & 12,5 \\
\hline \multicolumn{3}{|l|}{ Sexo del interlocutor de la iniciativa } \\
\hline Hombre & 51 & 44,0 \\
\hline Mujer & 35 & 30,2 \\
\hline dos o mas autores & 18 & 15,5 \\
\hline No especificado & 12 & 10,3 \\
\hline \multicolumn{3}{|l|}{ Grupo parlamentario que promueve la iniciativa } \\
\hline Grupo Parlamentario Popular & 50 & 43,1 \\
\hline Grupo Parlamentario Socialista & 48 & 41,4 \\
\hline Grupo Parlamentario Catalán (Convergència i Unió) & 9 & 7,8 \\
\hline Otros & 9 & 7,7 \\
\hline \multicolumn{3}{|l|}{ Toma de decisiones con impacto en las políticas } \\
\hline $\mathrm{Si}$ & 3 & 2,6 \\
\hline No & 113 & 97,4 \\
\hline \multicolumn{3}{|l|}{ Bloques temáticos } \\
\hline $\begin{array}{l}\text { Estrategias para dar atención en salud a la población } \\
\text { inmigrante }\end{array}$ & 29 & 25,0 \\
\hline Acceso a Servicios de salud & 28 & 24,1 \\
\hline Información estadística y epidemiológica & 23 & 19,8 \\
\hline Atención específica obstétrica y ginecológica & 14 & 12,1 \\
\hline Recursos disponibles para atención a la población & 14 & 12,1 \\
\hline Requerimientos de pruebas y medidas de protección & 3 & 2,6 \\
\hline Salud laboral e inmigración & 2 & 1,8 \\
\hline Formación profesional en temas de salud & 1 & 0,9 \\
\hline Convenios interinstitucionales en salud & 1 & 0.9 \\
\hline
\end{tabular}


Se presenta una primera descripción de un fenómeno político en España como un punto de partida para nuevas investigaciones que aborden los argumentos y posicionamientos de los diferentes grupos políticos. Las limitaciones de este estudio estarían relacionadas con la gran proporción de IP difíciles de clasificar por su heterogeneidad. Por ello, el grupo investigador seleccionó aquellas que por consenso fueran más claras y explicativas del tema en cuestión. Por otra parte, algunos bloques temáticos en los que se han clasificado las IP son de carácter general, como las estadísticas o las políticas y estrategias. En este sentido sería de gran interés indagar de forma complementaria acerca de los contenidos específicos en otras fuentes de información y de acción política.

La información estadística sobre el fenómeno migratorio constituye un recurso importante para la acción política, interactuando además con temas relevantes como la inserción en el mercado de trabajo de la población inmigrante $(2,11)$. Además, el aumento de las IP en los últimos años parece coincidir con el incremento de la proporción de inmigrantes de países en desarrollo y población inmigrante latinoamericana que inició procesos de regularización en España (1-3). Por otra parte, las IP también muestran el debate sobre políticas de control de fronteras con el fin de registrar la llegada de inmigrantes en condiciones de irregularidad.

La preocupación política en torno a la salud de la población inmigrante está basada fundamentalmente en el acceso a los servicios de salud. La indudable condición de derecho humano que tiene la salud y el hecho de que el sistema sanitario español sea universal en la atención contribuyen a que el debate político sea prioritario y que los servicios sanitarios constituyan un contexto fundamental en el que intervenir políticamente $(7,11,22,23)$. También es destacable que el debate sobre la salud de las mujeres inmigrantes se enfoque a la atención obstétrica (13), teniendo que cuenta que gran parte de los nacimientos ocurridos en España en los últimos años provienen de madre extranjera (1).

Las necesidades inmediatas de atención sanitaria que requiere la población inmigrante centran el debate político en la adecuación del sistema sanitario español a esta nueva coyuntura social (23). Con respecto a la atención sanitaria en la Constitución Española y en la Ley de extranjería, se discute la importancia de garantizar la asistencia al menor de edad, a la embarazada, la atención de urgencias y la protección del trabajador en el marco de la seguridad social junto a otros decretos reglamentarios generales y por Comunidades Autónomas (4-7). Sin embargo, parece existir una ausencia de interés político sobre aspectos que 
consideren la inmigración y su impacto en la salud dentro de un marco más amplio de determinantes de la salud, como el propuesto por la Comisión de Determinantes Sociales de la Organización Mundial de la Salud (24).

Aunque el debate político español comienza a abarcar temas importantes para la inmigración y la salud, sería pertinente una aproximación más integral, caracterizada por la reflexión sobre el contexto mundial, nacional y local en el que se da dicho proceso (25). En este sentido, la eficacia de las políticas públicas puede verse condicionada por el enfoque que se le dé al tema. Por ello, la investigación en inmigración y salud puede constituir una herramienta útil para analizar el fenómeno (26) y explorar, mediante metodologías como la cualitativa, la propia percepción de la población inmigrante respecto a su situación de salud (26-28) o identificar las consecuencias sobre la salud de la inserción en el mercado laboral $(14,28)$.

Finalmente, la ausencia de decisiones políticas sobre los temas debatidos en el parlamento constituye una barrera para la consolidación política y social de las acciones dirigidas a la población inmigrante. Además, confiere a dichas intervenciones un carácter puntual que puede ser susceptible a los cambios políticos en el gobierno. Es por ello que la toma de decisiones sería, por lo tanto, la vía adecuada para consolidar la acción política dirigida a asegurar la equidad en salud entre la población autóctona e inmigrante

Agradecimientos. Al Observatorio de Salud de la Mujer del Ministerio de Sanidad y Consumode España por el patrocinio del estudio "Informe sobre la Actividad Parlamentaria Española y la producción científica sobre aspectos relacionados con la salud de la población de mujeres y hombres Inmigrantes" que fue una primera aproximación al estudio del debate parlamentario sobre inmigración y salud en España. Este manuscrito es producto del proceso de formación de Andrés A. Agudelo-Suárez en el programa de Doctorado en Salud Pública, y será empleado como parte del material presentado para la disertación de la tesis doctoral en la Universidad de Alicante.

\section{REFERENCIAS}

1. Instituto Nacional Estadística. Explotación estadística del padrón. [Internet] Disponible en: http:// www.ine.es Consultado Agosto de 2007.

2. Arango J. La inmigración en España a comienzos del siglo XXI. En: Maldonado J. Informe sobre la situación demográfica en España. España: Fundación Fernando Abril Martorell; 2004. pp 161-186.

3. Ministerio de trabajo y asuntos sociales. Secretaría de Estado de Inmigración y Emigración. Extranjeros con tarjeta o autorización de residencia en vigor. [Internet] Disponible en: http://extranjeros.mtas.es. Consultado Agosto de 2007. 
4. Saura M. La prestación de asistencia sanitaria de la seguridad social a los extranjeros. Scripta Nova. Revista Electrónica de Geografía y Ciencias Sociales. Universidad de Barcelona 2001; 94. [Internet] Disponible en: http://www.ub.es/geocrit/sn-94-99.htm. Consultado Febrero de 2008.

5. Buchel F, Frick JR. Immigrants' economic performance across Europe. Does immigration policy matter? Population Research and Policy Review 2005; 24: 175-212.

6. Bruycker P. Regularización y política migratoria en Europa. Afers internacionals 2001; 53: 179187.

7. Martín I. La asistencia sanitaria de los extranjeros en España. Revista Derecho y Salud. 2002; 10: 197-218.

8. Roca C, Balanzó X. Enfermedades importadas en inmigrantes: mito y realidad. An Sist Sanit Navar 2006; 29 (Supl 1): 139-144.

9. Benítez RT, Llerena AP, López GR, Brugera MC, Lasheras L. Determinantes socioeconómicos en un colectivo de familias inmigrantes. An Pediatr (Barc) 2004; 60(1):9-15.

10. Moreno M. La asistencia al paciente inmigrante: un reto para los gestores sanitarios. Gestión Hospitalaria 2004; 15:85-92.

11. Ramos M, García R, Prieto MA, March JC. Problemas y propuestas de mejora en la atención sanitaria a los inmigrantes económicos. Gac Sanit 2001; 15(4): 320-326.

12. Pallás CR, De la Cruz J. Inmigración y salud infantil. Una perspectiva diferente. Rev Pediatr Aten Primaria 2003; 5: 9-16.

13. De la Torre J, Coll C, Coloma M, Martín JI, Padrón E, González NL. Control de gestación en inmigrantes. An Sist Sanit Navar 2006; 29 (Supl.1):49-61.

14. García AM, López-Jacob MJ, Agudelo-Suárez AA, Ruíz-Frutos C, Porthé V. Condiciones de trabajo y salud en inmigrantes (proyecto ITSAL): entrevistas a informantes clave. Gac Sanit 2009; 23 (2): 91-99.

15. Zapata R. Política del discurso sobre inmigración en España: discurso re-activo y discurso proactivo en los debates parlamentarios. Discurso y Sociedad. 2007; 1(2): 317-369.

16. Pastor P. El estilo político de la clase parlamentaria española: Propuesta metodológica para su estudio mediante análisis de contenido del Diario de Sesiones del Congreso de los Diputados (Comisiones). Empiria- Revista Metodológica de Ciencias Sociales. 2002; 5:109-142.

17. Vives-Cases C, Gil-González D, Carrasco-Portiño M, Álvarez-Dardet C. La violencia de género en la agenda del Parlamento español (1979-2004). Gac Sanit. 2006; 20(2):1428.

18. Umaña RA, Álvarez-Dardet Díaz C, Vives-Cases C. La opacidad de los acuerdos generales de bienes y servicios en España. Gac Sanit 2006; 20(3): 228-232.

19. Cambronero-Saiz B, Ruiz-Cantero M, Vives-Cases C, Carrasco-Portiño M. Abortion in Democratic Spain: The Parliamentary Political Agenda 1979-2004.Reprod Health Matters. 2007; 15 (29): 85-96.

20. García-Ferrando F, Ibáñez J, Alvira F. El análisis de la realidad social. Métodos y técnicas de investigación social. Madrid: Alianza Editorial; 2000.

21. Congreso de los Diputados. Buscador de Iniciativas Parlamentarias. [Internet] Disponible en: http://www.congreso.es. Consultado Julio de 2007.

22. Jansá JM, García P. Salud e inmigración: nuevas realidades y nuevos retos. Gac Sanit. 2004; 18 (Supl): 207-13.

23. Berra S, Elorza JM, Bartomeu N, Hausmann S, Serra-Sutton V, Rajmil L. Necesidades en salud y utilización de los servicios sanitarios en la población inmigrante en Cataluña. Revisión exhaustiva de la literatura científica. Barcelona: Agència d'Avaluació de Tecnologia i Recerca Mèdiques. CatSalut. Departament de Sanitat i Seguretat Social. Generalitat de Catalunya; 2004. 
24. World Health Organisation. Commission of Social Determinants of Health. A conceptual framework for action on social determinants of health. [Internet] Disponible en: http:// www.who.int/social_determinants/resources/csdh_framework_action_05_07.pdf. Consulado Junio de 2007.

25. MacPherson Douglas W, Gushulak Brian D, Macdonald Liane. Health and foreign policy: influences of migration and population mobility. Bull World Health Organ 2007; 85(3): 200-206.

26. Soriano R. Voces de mujeres desde la inmigración: Una comparativa entre el asentamiento de marroquíes en España y mexicanas en EEUU. Working Paper 133. San Diego. Center for Comparative Immigration Studies- University of California; 2006.

27. Agudelo-Suárez A, Ronda-Pérez E, Gil-González D, Vives-Cases C. Percepción sobre Condiciones de Trabajo y salud de la población inmigrante colombiana en Alicante, España. Rev. Salud Pública (Bogotá) 2008; 10 (1): 160-167.

28. Porthé V, Amable M, Benach J. La precariedad laboral y la salud de los inmigrantes en España: ¿Qué sabemos y qué deberíamos saber? Arch Prev Riesgos Labor 2007; 10 (1): 34-39. 